\title{
Renormalized Interactions of Interfaces, Membranes and Polymers
}

\author{
Reinhard Lipowsky \\ Sektion Physik der Universität München, Theresienstr. 37, D-8000 München 2, FRG \\ Received March 7, 1989; accepted April 11, 1989
}

\begin{abstract}
Wetting, adhesion, and adsorption phenomena are considered which involve the mutual interaction of low-dimensional objects such as interfaces, membranes, and polymers. In general, the direct interactions arising from intermolecular forces are strongly renormalized by thermally-excited shape fluctuations. This renormalization leads to a variety of unbinding transitions which exhibit complex critical behavior. The corresponding renormalization group flow is governed by a line of nontrivial fixed points and has a parabolic character.
\end{abstract}

\section{Introduction and outline}

Low-dimensional objects or manifolds such as interfaces, membranes, and polymers are usually soft and flexible and thus undergo thermally-excited shape fluctuations. In this paper, I will discuss the influence of these shape fluctuations on the mutual interactions between the manifolds. Such interactions govern various physical phenomena such as wetting, adhesion, and adsorption processes, see Section 2.

Quite generally, the shape fluctuations renormalize the direct interactions which arise from intermolecular forces. This renormalization acts to increase the repulsive part of the interaction. In fact, sufficiently strong fluctuations overcome the attractive part of the direct interactions and lead to phase transitions from bound to unbound states of the manifolds. For interfaces, membranes, and polymers, these unbinding transitions represent wetting, adhesion, and adsorption transitions, respectively.

Some insight into the renormalization by shape fluctuations can be obtained from simple scaling arguments. Thus, one may derive an effective fluctuation-induced interaction if the manifolds are viewed as ensembles of humps or blobs, see Sections 3 and 4. Superposition of this fluctuation-induced interaction and the direct interaction leads to the correct identification of several scaling regimes (or universality classes), see Section 5.

However, the critical behavior at the unbinding transition cannot be obtained, in general, by such a simple approach. Instead, one has to use genuine renormalization group (RG) methods which include shape fluctuations on many length scales. One then finds that the RG flow of the interactions is quite complex: it involves a whole line of nontrivial fixed points and has an unusual parabolic character, see Section 6 .

In this review, I will omit all technical details and try to emphasize the basic concepts which lead to a unified view of wetting, adhesion, and adsorption phenomena. The interested reader may find some of the missing details in Ref. [1].

\section{Wetting, adhesion, and adsorption phenomena}

A variety of physical phenomena is governed by the mutual interaction of interfaces, membranes, and polymers. The geometry of these phenomena is shown in Fig. 1.

Wetting phenomena occur when an interface between two macroscopic bulk phases, $\alpha$ and $\gamma$, contains a thin film or layer of a third phase, $\beta$, see Fig. 1(a). The intermediate $\beta$ layer is bounded by two interfaces, and its thickness is determined by the mutual interactions of these interfaces [2]. Such a geometry has been experimentally observed in many different systems including multilayer adsorption of small molecules, surfaces melting, and surface-induced disorder.

Adhesion of an oriented membrane onto an interface is shown in Fig. 1(b). Such a geometry underlies, e.g., the construction of biosensors where a lipid bilayer is "immobilized" onto a solid substrate. Likewise, the membrane in Fig. 1(b) can be a small segment of a large lipid vesicle which adheres to the interface. One may also replace this interface by a second membrane and, thus, consider the mutual interaction of two oriented membranes [3].

Adsorption of a polymer onto an interface or substrate is shown in Fig. 1(c). For good solvent conditions, the adsorbed polymer forms a random coil which is constrained by the substrate potential [4]. Such polymers are important, e.g., for the stabilization of colloidal suspension. In the context of biophysics, they serve as simple models for the macromolecules which are attached to biomembranes.

Finally, adsorption or binding of a crumpled membrane to an interface is shown in Fig. 1(d). Crumpled membranes are the 2-dimensional analogues of polymers. Such highly convoluted surfaces are present in microemulsions, i.e., in mixtures of water, oil and surfactant where they separate water and oil domains [3].

The low-dimensional objects or manifolds shown in Figs 1 (a)-(d) form bound states in the sense that their mean separation has a finite value. These states require the presence of some attractive interaction between the manifolds.

For a given shape of the interfaces, membranes, or polymers, their mutual interaction directly reflects microscopic intermolecular forces. This direct interaction consists of several short-ranged and long-ranged contributions: e.g., electrostatic interactions arising from the forces between charges molecules, van der Waals interactions as a result of dipole-dipole forces, or structural interactions which usually arise from short-range order within the solvent.

There is, in fact, a huge literature on intermolecular forces and the corresponding direct interaction [5]. However, it has been realized only recently that these interactions are strongly renormalized by shape fluctuations. Here, I will focus on thermally-excited fluctuations which increase the configurational entropy of the manifolds. 


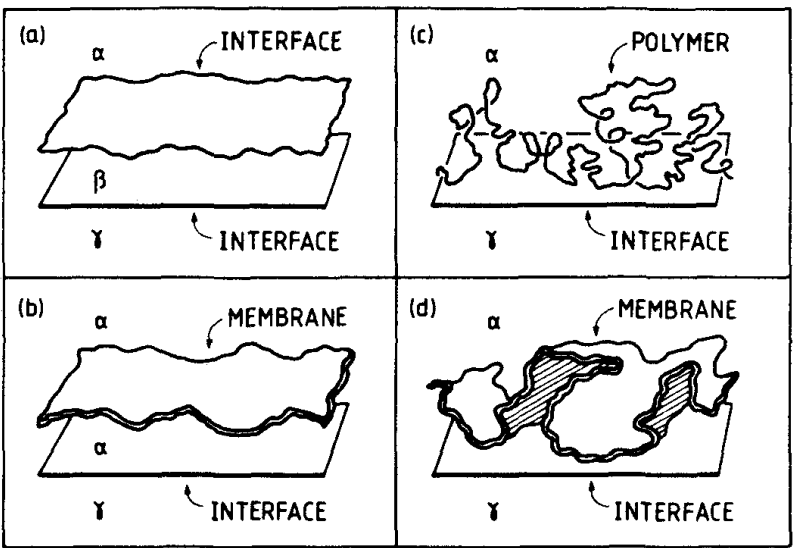

Fig. 1. (a) Geometry of wetting, surface melting and related phenomena; (b) adhesion of an oriented membrane; (c) adsorption of a polymer; and (d) adsorption of a crumpled membrane. Distinct thermodynamic phases are denoted by $\alpha, \beta$ and $\gamma$.

\section{Hump picture for oriented manifolds}

The fluctuating manifolds shown in Figs 1(a) and (b) are orientationally ordered, i.e., their normal vectors have a preferred direction. In general, each fluctuating manifold can be characterized by a persistence length, $\xi_{p}$, for its orientational order $[6,7]$. In this section, I will consider oriented manifolds which have a linear size $L \ll \xi_{p}$

The bound state of an oriented manifold can be viewed as an ensemble of humps [8,9]. On large scales, these humps have a longitudinal extension set by the correlation length, $\xi_{\|}$, for transverse displacements, and a typical roughness

$\xi_{\perp} \sim \xi_{\|}^{\zeta} \quad$ with $\zeta>0$.

For a free or unbound manifold of longitudinal extension $L_{\|}$, this relation becomes $L_{\perp} \sim L_{\|}^{\zeta}$ where $L_{\perp}$ is the overall amplitude of the transverse fluctuations.

For an interface, the exponent $\zeta$ depends on the spatial dimensionality, $d$, of the system and on the nature of the two adjacent phases which can be fluids, periodic crystals, or quasi-crystals; it also depends on the presence of quenched disorder which I will not discuss here [1]. For an oriented membrane, the exponent $\zeta$ depends on the internal membrane structure which can be fluid, crystalline or hexatic [1]. For example, one usually has $\zeta=1 / 2$ for interfaces in $\mathrm{d}=1+\mathrm{I}$, and $\zeta=1$ for fluid membranes in $d=2+1$

Since $\xi_{\|}$is the correlation length for transverse displacements, different humps are essentially uncorrelated. Thus, a bound manifold with projected area, $L_{\|}^{d_{\|}}$, may be viewed as an emsemble of $\left(L_{\|} / \xi_{\|}\right)^{d_{\|}}$independent humps. Here $d_{\|}$is the intrinsic or parallel dimensionality of the manifold which is embedded in $d=d_{\|}+d_{\perp}$ dimensions [10]. The thermal free energy of each hump should be $\sim T$ as suggested by the equipartition theorem [11]. Therefore, the thermal free energy, $F_{\mathrm{FL}}$, of the whole manifold can be estimated by

$F_{\mathrm{FL}} \simeq T\left(L_{\|} / \xi_{\|}\right)^{d_{\|}}$,

and the thermal free energy per unit projected area is

$V_{\mathrm{FL}} \equiv F_{\mathrm{FL}} / L_{\|}^{d_{\|}} \sim 1 / \xi_{\|}^{d_{\|}} \sim 1 / \xi_{\perp}^{d_{\|} / \xi}$.

In addition, one may assume that the mean distance, $l$, between the two interacting manifolds is $\sim \xi_{1}$. It turns out that this assumption is fulfilled as long as the direct interac- tion is sufficiently short-ranged, see Section 5 below. In these cases, one has $[8,9]$

$V_{\mathrm{FL}}(l) \sim 1 / l^{\tau} \quad$ with $\tau \equiv d_{i /} / \zeta$.

This expression can be regarded as a fluctuation-induced repulsion acting between two oriented manifolds.

Consider again the geometry shown in Fig. 1 where one of the two interacting manifolds is a flat and, thus, oriented interface. If the other manifold is characterized by $\zeta<1$, its persistence length $\xi_{p}$ is infinite, and the manifold will stay oriented on arbitrarily large scales even in the unbound state. On the other hand, for $\zeta \geqslant 1$, the persistence length $\xi_{p}$ is finite: the unbound manifold will loose its orientation and start to crumple as soon as its linear size $L>\xi_{p}$

\section{Blob picture for crumpled manifolds}

Bound states of crumpled manifolds are displayed in Figs 1(c) and 1(d). For an adsorbed layer of thickness $l$, the large scale configurations can be viewed as blobs of linear size $l$ [12-14]. Each blob contains a manifold piece with total area $\sim\left(N_{l} a\right)^{d_{1}}$ consisting of $N_{f}^{d_{\|}}$segments of linear size $a$. Here, $a$ is a smalldistance cutoff for the crumpled membrane, and $d_{\|}$is its intrinsic dimensionality as before. The linear size of the blobs and the segment number within each blob satisfy

$l \sim N_{j}^{v} \quad$ with $v \leqslant 1$.

For a free or unbound manifold, this relation becomes $R_{\mathrm{G}} \sim N^{v}$ where $R_{\mathrm{G}}$ is the radius of gyration and $N^{d_{\|}}$is the total monomer number $\sim$ total area of the manifold.

For polymers with $d_{\|}=1$, the exponent $v$ depends on $d_{\perp}=d-d_{\|}$, on its linear or branched geometry, and on the presence or absence of electric charges. For crumpled membranes with $d_{\|}=2$, this exponent is believed to depend also on the internal membrane structure. For example, one has [15] $v \simeq 3 / 5$ for linear polymers in $d=1+2$ [16].

It is again plausible to assume that correlations between different blobs can be neglected. Then, a crumpled manifold with $N^{d_{\|}}$monomers consists of $\left(N / N_{l}\right)^{d_{\|}}$independent blobs. The thermal free energy stored within each blob should again be $\sim T$ because of the equipartition theorem. Therefore, the thermal free energy, $F_{\mathrm{FL}}$, of the crumpled manifold can be estimated by

$F_{\mathrm{FL}} \simeq T\left(N / N_{l}\right)^{d_{i !}}$.

Then, the thermal free energy per unit intrinsic area is [13]

$V_{\mathrm{FL}} \equiv F_{\mathrm{FL}} /(a N)^{d_{\|}} \sim 1 / N_{l}^{d_{\|}} \sim 1 / l^{\tau} \quad$ with $\tau \equiv d_{\|} / \nu$.

\section{Different scaling regimes for the interactions between manifolds}

In the two previous sections, the excess free energy arising from thermally-excited shape fluctuations of a bound manifold has been estimated in a heuristic way. This led to an effective fluctuation-induced repulsion, $V_{F L}$, which scales as

$V_{\mathrm{FL}}(l) \sim 1 / l^{\tau} \quad$ with $\tau=d_{\|} / \zeta \quad$ and $\tau=d_{\|} / v$

for oriented and crumpled manifolds, respectively [17, 18].

Now, this excess free energy will be compared with the direct interaction arising from intermolecular forces such as van der Waals, electrostatic or structural forces. For each shape or configuration of the bound manifold, these forces 
give rise to an interaction free energy, $V_{\mathrm{DV}}$, which represents the excess free energy of the given bound state as compared with the unbound state of the manifold. For oriented and crumpled manifolds, $V_{\mathrm{Dl}}$ is defined to be the interaction free energy per unit projected and per unit intrinsic area, respectively.

For the geometries as shown in Fig. 1, the direct interaction, $V_{\mathrm{Dl}}$, depends on the coordinate $z$ which measures the distance of the fluctuating manifold from the flat interface. Then, the interaction free energy of the bound manifold can be estimated by

$\left\langle\boldsymbol{V}_{\mathrm{DI}}(z)\right\rangle \sim V_{\mathrm{DI}}(l) \quad$ with $l \equiv\langle z\rangle$.

Now, consider a certain pair of manifolds characterized by the fluctuation-induced interaction $V_{\mathrm{FL}} \sim 1 / l^{\tau}$ and, thus, by a certain value of $\tau$. Then, the space of all possible direct interactions, $\boldsymbol{V}_{\mathrm{Dl}}$, between these manifolds consists of four different scaling regimes. These regimes can be identified by a simple comparison or superposition of $\boldsymbol{V}_{\mathrm{FL}}$ and $\boldsymbol{V}_{\mathrm{DI}}$ both for oriented [8] and for crumpled [13] manifolds. The four regimes are defined as follows:

(i) The mean field (MF) regime characterized by

$$
V_{\mathrm{DR}}(l) \gg V_{\mathrm{FL}}(l) \sim 1 / l^{\mathrm{t}} \quad \text { for large } l
$$

where $V_{\mathrm{DR}}$ represents the repulsive part of $V_{\mathrm{DI}}$. In this situation, the mean separation, $l$, of the manifolds is not affected by shape fluctuations $[2,19]$. This regime contains, e.g., wetting [20] or surface melting [21] in 3-dimensional systems when governed by long-ranged van der Waals forces;

(ii) The weak-fluctuation (WFL) regime is defined by

$V_{\mathrm{DR}}(l) \ll V_{\mathrm{FL}}(l) \sim 1 / l^{\tau} \ll V_{\mathrm{DA}}(l) \quad$ for large $l$

where $V_{\mathrm{DA}}$ represents the attractive part of $V_{\mathrm{DI}}[2,19,13]$. The critical behavior within this regime can be obtained in a rather simple way by minimization of $V_{\mathrm{FL}}+V_{\mathrm{Dr}}$. The WFL regime contains, e.g., complete wetting or edge melting in 2 -dimensional systems [1,22], and adsorption of polymers or crumpled membranes by a weak gravitational field [13].

In addition, the superposition Ansatz, $V_{\mathrm{FL}}+V_{\mathrm{Di}}$, indicates two nontrivial scaling regimes even though it does not give a correct description for the critical behavior within these regimes:

(iii) The intermediate-fluctuation (IFL) regime with

$\mid V_{\mathrm{DI}}(l) \sim V_{\mathrm{FL}}(l) \sim 1 / l^{\tau} \quad$ for large $l$.

In this case, the superposition Ansatz indicates that the critical behavior depends both on the long-ranged tail $\sim 1 / l^{\tau}$ and on the short-ranged part of $\boldsymbol{V}_{\mathrm{DI}}(l)$. In fact, this regime contains three different subregimes $[13,23]$ with rather complex critical behavior, see Section 6 below; and

(iv) Finally, the strong-fluctuation (SFL) regime characterized by sufficiently short-ranged interactions with

$\mid V_{\mathrm{DI}}(l) \ll V_{\mathrm{FL}}(l) \sim 1 / l^{\star} \quad$ for large $l$.

In this regime, the superposition Ansatz predicts a first-order transition while the transition is, in fact, continuous and governed by universal critical exponents. These exponents are known in some but not in all cases of physical interest [24-27]. The SFL regime contains, e.g., adhesion transitions of fluid membranes which have been recently observed in experiments [28].

The above classification scheme must be modified for wetting phenomena in $d=3$ governed by $V_{\mathrm{FL}} \sim \exp \left(-c \xi_{\perp}^{2}\right)$ and short-range direct interactions [1]. Indeed, this case with $l \sim \xi_{\perp}^{2}$ is expected to exhibit rather subtle fluctuation effects. On the other hand, computer simulations are, so far, consistent with mean-field behavior over the accessible temperature range $[29,30]$. Thus, recent experiments on surface melting of $\mathrm{Pb}[31,32]$ and on surface-induced disorder of $\mathrm{CuAu}[33,34]$ are likely to probe mean-field behavior as well $[35,36]$.

\section{Parabolic flow of the renormalized interactions}

The different scaling regimes as given by eqs. (5.1)-(5.4) have been obtained from the rather heuristic superposition of fluctuation-induced and direct interactions. This classification is, however, fully confirmed by more systematic methods. On the other hand, the superposition Ansatz fails for the critical behavior within the nontrivial regimes as given by equations (5.3) and (5.4). As explained below, a functional renormalization group (RG) approach reveals that the critical behavior within these regimes is, in fact, rather unusual.

\subsection{Effective Hamiltonian}

To proceed, let us again consider the geometry displayed in Figs 1(a) and (b). As before, the shape of the fluctuating manifold will be parametrized by $z=z(x)$ where $x$ is a $\boldsymbol{d}_{\|}$-dimensional coordinate parallel to the flat interface (with $\left.d_{\|}=d-1\right)$. The effective Hamiltonian of the fluctuating manifold has the generic form

$\mathrm{H}\{z\}=\int \mathrm{d}^{d_{11}} x\left\{\frac{1}{2} K\left(\nabla^{n} z\right)^{2}+V_{\mathrm{DI}}[z(\boldsymbol{x})]\right\}$

with

$n \equiv \zeta+d_{\|} / 2$

and a small-distance cutoff, a, is implicitly contained. The first term in eq. (6.1) describes the elastic energy associated with the shape fluctuations while the second term represents the direct interactions of the manifolds. For interfaces and membranes, the elastic constant, $\boldsymbol{K}$, is the interfacial stiffness and the bending rigidity, respectively. As usual, the statistical weight for the manifold configurations is given by the Boltzmann factor, $\exp [-\mathrm{H}\{z\} / T]$

A Hamiltonian of the form (6.1) can also be used for the adsorption of crumpled manifolds, compare Figs 1(c) and (d), provided one ignores the selfavoidance constraint and allows for selfintersections [13]. The shape of such an ideal (or phantom) manifold will be parametrized by $\left[x_{1}(s), \ldots\right.$, $\left.x_{d-1}(s), z(s)\right]$ where $s$ is an appropriate intrinsic coordinate. Then, the effective Hamiltonian for the crumpled (but ideal) manifold becomes [13]

$$
\begin{aligned}
& \mathbf{H}\left\{x_{1}, \ldots, z\right\} \\
& \quad=\int \mathrm{d}^{d_{11}} s\left\{\frac{1}{2} K \sum_{\alpha}\left(\nabla x_{\alpha}\right)^{2}+\frac{1}{2} K(\nabla z)^{2}+V_{\mathrm{D} 1}[z(s)]\right\}
\end{aligned}
$$

In this case, the elastic constant, $\boldsymbol{K}$, is entropically generated and $\boldsymbol{K} \sim \boldsymbol{T} / \boldsymbol{a}^{\boldsymbol{d}_{\boldsymbol{1}}}$. For such a model, the $\boldsymbol{x}$ - and $z$-coordinate decouple and the effective Hamiltonian for $z(s)$ has the same form as eq. (6.1) with $n=1$

The statistical properties which follow from the models as given by eq. (6.1) or (6.3) can be studied by a variety of theoretical methods. So far, the most useful approach has been a functional $\mathrm{RG}$ which represents an extension of Wilson's approximate recursion relations [37, 38]. 


\subsection{Lines of renormalization group fixed points}

For infinitesimal rescaling factor $b \rightarrow 1+\Delta t$, this functional $\mathrm{RG}$ leads to the nonlinear flow equation [38]

$$
\begin{aligned}
\partial V / \partial t= & d_{\|} V+\left(d_{\|} / \tau\right) z \partial V / \partial z \\
& +\frac{1}{2} v \ln \left[1+\left(a_{\perp}^{2} / v\right) \partial^{2} V / \partial z^{2}\right]
\end{aligned}
$$

for the renormalized interactions $V(z \mid t)$ with the scale factors

$v \sim T / a^{d_{\sharp}} \quad$ and $\quad a_{\perp}^{2} \sim(T / K) a^{2 d_{\|} / \tau}$,

where $d_{\|} / \tau=\zeta$ and $d_{\|} / \tau=v=1-d_{\|} / 2$ for oriented and ideal crumpled manifolds, respectively. The "initial" interaction at $t=0$ is given by the direct interaction: $V(z \mid t=0)=V_{\mathrm{DI}}(z)$

Now consider the space of all interactions $V(z)$ which decay to zero for large $z$ at least as $\sim 1 / z^{z}$. This function space contains both the strong-fluctuation (SFL) and the intermediate-fluctuation (IFL) regime defined by eqs. (5.4) and (5.3). A detailed study of the RG transformation as given by eq. (6.4) reveals that this space is governed, for each value of $\tau$, by a whole line of RG fixed points, $V^{*}(z)$, with $\partial V^{*} / \partial t=0$ [39].

It is convenient to use the dimensionless variables

$y \equiv\left(2 d_{\|} / \tau\right)^{1 / 2} z / a_{\perp} \sim z(K / T)^{1 / 2} / a^{d_{\|} / \tau}$

and

$U \equiv 2 d_{\|} V / \tau v \sim V a^{d_{\|}} / T$.

Then, the line of fixed points, $U^{*}(y) \sim V^{*}(z)$, is characterized, for large $y \sim z$, by the asymptotic behavior [39]

$U^{*}(y) \approx \varrho_{\mathrm{L}}\left(\varrho_{\mathrm{s}}\right) / y^{t}+\varrho_{\mathrm{s}} y^{\tau-1} \exp \left(-y^{2} / 2\right)$

The amplitude $\varrho_{\mathrm{L}}$ of the long-range power-law tail is found to be convex downwards as a function of $\varrho_{\mathrm{S}}$ and to have a unique minimum at $\varrho_{\mathrm{S}}=\varrho_{\mathrm{S}}^{*}$ with

$\varrho_{\mathrm{L}}\left(\varrho_{\mathrm{S}}\right) \approx \varrho_{\mathrm{S}}^{*}+\frac{1}{2} R_{2}\left(\varrho_{\mathrm{S}}-\varrho_{\mathrm{S}}^{*}\right)^{2}$

and $R_{2}=R_{2}(\tau)>0$ [40].

Close to this minimum, the RG flow within the $\propto$-dimensional interaction space can be reduced to the flow within the 2-dimensional $\left(\varrho_{\mathrm{L}}, \varrho_{\mathrm{S}}\right)$-space as given by [40]

$\partial \varrho_{\mathrm{L}} / \partial t=0$

and

$\partial \varrho_{\mathrm{S}} / \partial t=c_{1}\left(\varrho_{\mathrm{L}}-\varrho_{\mathrm{L}}^{*}\right)-c_{2}\left(\varrho_{\mathrm{s}}-\varrho_{\mathrm{S}}^{*}\right)^{2}$

with $c_{1}, c_{2}>0$. The RG trajectories of this flow are shown in Fig. 2(a).

\subsection{Parabolic versus hyperbolicflow}

The parabola displayed in Fig. 2(a) represents the line of RG fixed points. This line has two branches: $\varrho_{\mathrm{s}}=\bar{\varrho}_{0}$ and $\varrho_{\mathrm{s}}=\bar{\varrho}_{\mathrm{c}}$ which merge at the point $\left(\varrho_{\mathrm{L}}, \varrho_{\mathrm{S}}\right)=\left(\varrho_{\mathrm{L}}^{*}, \varrho_{\mathrm{S}}^{*}\right)$. The physical interpretation of these branches will be explained in the next section. First, I will discuss the parabolic character of the RG flow.

It is instructive to change variables according to $\boldsymbol{g}_{1} \equiv$ $c_{2}\left(\varrho_{\mathrm{S}}-\varrho_{\mathrm{S}}^{*}\right)$ and $g_{2} \equiv g_{1}^{2}-c_{1} c_{2}\left(\varrho_{\mathrm{L}}-\varrho_{\mathrm{L}}^{*}\right)$. Then, the flow equations (6.10) and (6.11) become

$\partial g_{1} / \partial t=-g_{2}$ and $\partial g_{2} / \partial t=-2 g_{1} g_{2}$.

Such a recursion relation has been previously considered by

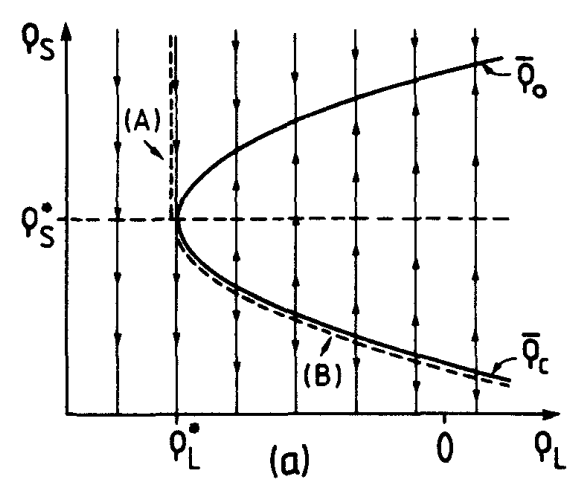

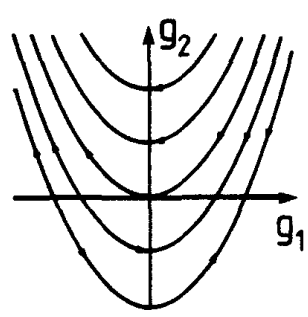

(b)

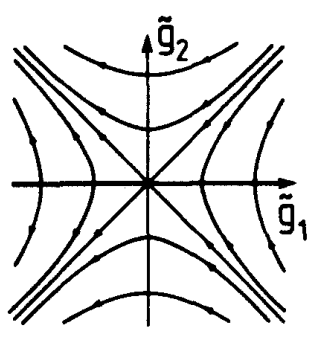

(c)
Fig. 2. (a) Renormalization group (RG) flow within the 2-dimensional $\left(\varrho_{\mathrm{L}}, \varrho_{\mathrm{S}}\right)$-space. The separatrix between bound and unbound states consists (A) of the unique RG trajectory which is mapped onto $\left(\varrho_{\perp}^{*}, \varrho_{\mathrm{S}}^{*}\right)$, and (B) of the lower branch, $\bar{\varrho}_{\mathrm{c}}$, of the line of fixed points; (b) equivalent parabolic flow: and (c) distinct hyperbolic flow.

Wegner [41]. Within the $\left(g_{1}, g_{2}\right)$-space, the RG trajectories are parabola given by $g_{2}=g_{1}^{2}+$ const., see Fig. 2(b). The line of fixed points now lies on top of the $g_{1}$-axis with $g_{2}=0$

The parabolic flow shown in Fig. 2(b) must be distinguished from the well-known Kosterlitz-Thouless flow as displayed in Fig. 2(c) [42]. The corresponding recursion relation is given by

$\partial \tilde{g}_{1} / \partial t=-\tilde{g}_{2}^{2}$ and $\partial \tilde{g}_{2}^{2} / \partial t=-\tilde{g}_{1} \tilde{g}_{2}$.

This Kosterlitz-Thouless RG also leads to a line of fixed points at $\tilde{g}_{2}=0$, as shown in Fig. 2(c), but the RG trajectories are hyperbola given by $\tilde{g}_{2}^{2}=\tilde{g}_{1}^{2}+$ const. rather than parabola.

\subsection{Phase diagram and critical behavior}

The parabolic RG flow shown in Fig. 2(a) has the following interpretation. All parameter values which flow into the upper branch, $\bar{\varrho}_{0}$, of the line of fixed points correspond to unbound states of the manifolds. All parameter values which are mapped under the RG to large negative values of $\varrho_{\mathrm{s}}$ corresponds to bound states of the manifolds. The separatrix between these two regions of the $\left(\varrho_{\mathrm{L}}, \varrho_{\mathrm{S}}\right)$-space is the locus of unbinding transitions. This locus consists (A) of the unique RG trajectory which flows into the special point $\left(\varrho_{\mathrm{L}}^{*}, \varrho_{\mathrm{S}}^{*}\right)$ and (B) of the lower branch, $\bar{\varrho}_{\mathcal{E}}$, of the line of fixed points.

All states with $\varrho_{\mathrm{L}}<\varrho_{\mathrm{L}}^{*}=\varrho_{\mathrm{L}}^{*}(\tau)$ are bound states. This implies, via eqs. (6.6) and (6.7), that a direct interaction, $V_{\mathrm{DI}}(z) \approx R_{\mathrm{L}} / z^{\tau}$, binds the manifolds together provided

$R_{\mathrm{L}}<-c T(T / K)^{i / 2}$

where $c=c(\tau)$ is a numerical coefficient. This property can be understood from the heuristic approach described in 
Section 5. Indeed, the superposition of fluctuation-induced and direct interactions leads to $V_{\mathrm{FL}}(l)+V_{\mathrm{DI}}(l) \approx\left[\bar{c} T(T / K)^{z / 2}+\right.$ $\left.R_{\mathrm{L}}\right] / l^{r}$ with $l \equiv\langle z\rangle$ (the $T$-dependent prefactor of $V_{\mathrm{FL}}(l)$ has been omitted in Sections 3-5). Thus, within this simple approach, the fluctuation-induced repulsion cannot overcome the attractive part of the direct interaction if $R_{\mathrm{L}}<$ $-\bar{c} T(T / K)^{\tau / 2}$ which is identical with eq. (6.14) apart from the numerical coefficients.

For a direct interaction $V_{\mathrm{DI}}(z)=R_{\mathrm{L}} / z^{\tau}$ with fixed $R_{\mathrm{L}}<0$ the manifolds undergo an unbinding transition as $T$ approaches $T_{*}$ from below with $c T_{*}\left(T_{*} / K\right)^{\tau / 2}=\left|R_{\mathrm{L}}\right|$. The corresponding temperature trajectory in the $\left(\varrho_{\mathrm{L}}, \varrho_{\mathrm{S}}\right)$-space intersects part (A) of the separatix with $\varrho_{\mathrm{L}}=\varrho_{\mathrm{L}}^{*}$. In this case, integration of the $\mathrm{RG}$ flow as given by eqs. (6.10) and (6.11) leads to the critical behavior [40]

$$
l \sim \exp \left[2 \pi / \bar{\omega} \sqrt{\varrho_{\mathrm{L}}^{*}-\varrho_{\mathrm{L}}}\right] \sim \exp \left[\text { const. } / \sqrt{T_{*}-T}\right]
$$

for the mean separation, $l$, of the manifolds with $\bar{\omega}=\bar{\omega}(\tau)$ [43]. Thus, the unbinding transition in subregime (A) is of infinite order.

On the other hand, consider a direct interaction of the more general form $V_{\mathrm{DI}}(z)=R_{\mathrm{L}} / z^{\tau}+R_{\mathrm{S}} / z^{p}$ with $p>\tau$ Then, the temperature trajectory can intersect part (B) of the separatrix with $\varrho_{\mathrm{L}}>\varrho_{\mathrm{L}}^{*}$. In this case, one obtains the power law behavior $[39,40]$

$$
l \sim\left(T_{*}-T\right)^{-\psi} \quad \text { with } \psi \approx 1 / \bar{\omega} \sqrt{\varrho_{\mathrm{L}}-\varrho_{\mathrm{L}}^{*}}
$$

for small $\varrho_{\mathrm{L}}-\varrho_{\mathrm{L}}^{*}$, and the critical exponent $\psi$ is found to depend on the amplitude $\varrho_{\mathrm{L}}$ of the long-ranged tail and, via $\bar{\omega}$ and $\varrho_{L}^{*}$, on $\tau$. Thus, subregime (B) is characterized by unbinding transitions of second order but with non-universal critical exponents.

Subregime (B) contains the case $\varrho_{\mathrm{L}}=R_{\mathrm{L}}=0$ which corresponds to the SFL regime with $\left|V_{\mathrm{DI}}(z)\right| \ll 1 / z^{\mathrm{t}}$ for large $z$, see Section (5.4). In this case, the RG considered here leads to a universal value for the critical exponent $\psi$ which is predicted to depend only on $\tau$ [44]. If one ignores possible higher-order terms in eq. (6.11), extrapolation of eq. (6.16) leads to the estimate

$\psi \simeq 1 / \bar{\omega} \sqrt{\left|\varrho_{\mathrm{L}}^{*}\right|}$

for the SFL regime. It turns out that higher-order terms must, in fact, be absent in an exact RG flow for $\tau=2$. This follows by comparison with exact results for wetting in $d=1+1$ [23]. In this case, the estimate (6.17) is indeed quite good: the $\mathrm{RG}$ transformation (6.4) leads to $[40] \bar{\omega} \simeq 1.16$ and $\varrho_{\mathrm{L}}^{*} \simeq 0.73$ for $\tau=2$ and, thus, to $\psi \simeq 1.01$ via eq. (6.17) which must be compared with the exact value $\psi=1$ for wetting in $d=1+1$

For large positive values of $\varrho_{L}$, one should enter a third subregume (C) which is, however, not correctly described by the $\mathrm{RG}$ approach used here. In this subregime, the continuous unbinding transition is preempted by a discontinuous transition: the transition then exhibits a jump in the first derivative of the free energy. Indeed, the free energy, $f_{s}$, scales as [45]

$f_{\mathrm{s}} \sim 1 / l^{\tau} \sim\left(T_{*}-T\right)^{\tau \psi}$

for $T<T_{*}$ with $\psi=\psi\left(\varrho_{\mathrm{L}}\right)$, compare eq. (6.16). Therefore, the transition becomes discontinuous for $\varrho_{\mathrm{L}}>\varrho_{\mathrm{L}}^{* *}$ where $\varrho_{\mathrm{L}}^{* *}$ satisfies $\tau \psi\left(\varrho_{\mathrm{L}}^{* *}\right)=1$. However, these first-order transitions are anomalous since they still exhibit nontrivial scaling properties $[23,39]$ : the probability distribution, $P_{*}(l)$, for the mean separation $l$ of the manifolds has a power law tail $\sim 1 / l^{\mu}$ for large $l$. For $\tau=2$, one has $\mu=\bar{\omega}(2) \sqrt{\varrho_{\mathrm{L}}-\varrho_{\mathrm{L}}^{*}}-1$ with $\bar{\omega}(2) \sqrt{\left|\varrho_{\mathrm{L}}^{*}\right|}=1$. This $\varrho_{\mathrm{L}}$-dependence of $\mu$ is related to the fact that, for $\tau=2$, the exact RG flow is parabolic for all values of $\varrho_{\mathrm{L}}$. It is not obvious how $\mu$ depends on $\varrho_{\mathrm{L}}$ if one has higher-order corrections in eq. (6.11) as expected for $\tau \neq 2$

\subsection{Related phenomena}

Finally, I want to argue that the RG flow and, thus, the phase diagram shown in Fig. 2(a) should apply to unbinding phenomena in general. In particular, these features should apply (i) to adsorption of selfavoiding crumpled manifolds, and (ii) to wetting in the presence of quenched disorder.

As mentioned, the branch $\bar{\varrho}_{0}$ of the line of fixed points, compare Fig. 2(a), corresponds to unbound states. Such states of the manifolds are scale invariant. For an oriented manifold parametrized by $z(\boldsymbol{x})$, the associated scale transformation has the form $\boldsymbol{x} \rightarrow \boldsymbol{x} / b$ and $z \rightarrow z / b^{5}$ with rescaling factor $b>1$. Likewise, crumpled manifolds with $\left[x_{1}(s), \ldots, z(s)\right]$ are invariant under $s \rightarrow s / b$ and $z \rightarrow z / b^{r}$ When such a scale transformation is applied to the direct interaction, one obtains $V^{\prime}(z)=b^{d_{\sharp}} V\left(b^{d_{\|}^{\prime} z} z\right)$ or, for $b \rightarrow 1+\Delta t$,

$\partial V / \partial t=d_{\|} V+\left(d_{\|} / \tau\right) z \partial V / \partial z$

with $d_{\|} / \tau=\zeta$ or $v$, i.e., the first two terms of the full recursion relation as given by eq. (6.4). The linear transformation (6.19) already leads to a line of fixed points given by $V^{*}(z) \sim$ $U^{*}(y)=\varrho_{\mathrm{L}} / y^{\tau}$, which approximates the branch $\bar{\varrho}_{0}$ for large positive values of $\varrho_{\mathrm{L}}$, compare Fig. 2(a).

On the other hand, for large negative values of $\varrho_{L}$, there should be no unbound states and, thus, no fixed points, see Section 6.14. Therefore, the line of fixed points should be restricted to $\varrho_{\mathrm{L}}>\varrho_{\mathrm{L}}^{*}>-\infty$. Furthermore, there must be a second branch, $\tilde{\varrho}_{c}$, which gives the separatrix for the unbinding transitions. This latter branch should exhibit one relevant perturbation while the branch for the unbound states should be completely stable. These properties, together with the usual assumption that the RG transformation has a smooth dependence on the physical parameters, seems to imply that the RG flow has the topology shown in Fig. 2(a).

\section{Summary}

In summary, the direct interactions of interfaces, membranes and polymers can be divided into several scaling regimes or universality classes (Section 5). If the interactions are sufficiently short-ranged, one enters two nontrivial regimes, the intermediate-fluctuation and the strong-fluctuation regime. These regimes are governed by a line of nontrivial renormalization group (RG) fixed points and exhibit an unusual. RG flow which has a parabolic character (Section 6). Such a flow has been explicitly derived for oriented and for ideal crumpled manifolds but should be a generic feature of unbinding phenomena. The various regimes are accessible to experiments on wetting, adhesion, and adsorption.

\section{Acknowledgements}

I thank Artur Baumgärtner, Frank Jülicher, Daniel Kroll, Thomas Nattermann, and Barbara Zielinska for stimulating interactions, and acknowledge support by the Deutsche Forschungsgemeinschaft via the SFB 266. 


\section{References}

1. Lipowsky, R., Random Fluctuations and Pattern Growth. (Edited by H. E. Stanley and N. Ostrowsky). Kluwer Academic Publishers, Dordrecht (1988) (and references therein).

2. See, e.g., Lipowsky, R., Phys. Rev. Lett, 52, 1429 (1984); Phys. Rev. B32, 1731 (1985); and Habilitations-Schrift, Ludwig-MaximiliansUniversität München (1987) (distributed by KFA Jülich as Jül-Spez438).

3. See, e.g., Physics of Amphiphilic Layers (Edited by J. Meunier, D. Langevin, and N. Boccara) Springer Proc. In Physics, Vol. 21 Springer-Verlag (1987)

4. See, e.g., de Gennes, P. G., Scaling Concepts in Polymer Physics Cornell University Press, New York (1979).

5. See, e.g., Israelachvili, J. N., Intermolecular and Surface Forces. (Academic (1985).

6. For membranes, the concept of a persistence length was introduced by de Gennes, P. G. and Taupin, D., J. Phys. Chem. 86, 2294 (1982).

7. In order to give a precise definition of the persistence length, $\xi_{p}$ consider the unbound state of the manifold and define the correlation function, $C_{n}(s) \equiv\langle\hat{n}(s) \cdot \hat{\boldsymbol{n}}(\mathbf{0})\rangle$, of its normal where $s$ is an intrinsic $\boldsymbol{d}_{\|}$-dimensional coordinate. The persistence length is finite or infinite if $C_{n}(s)$ decays to zero or to a finite value for large $s$, respectively.

8. For wetting, see Lipowsky, R. and Fisher, M. E., Phys. Rev. Lett. 56, 472 (1986); Phys. Rev. B36, 2126 (1987)

9. For adhesion of membranes, see Helfrich, W., Naturforschung, Z., 33a, 305 (1978); Lipowsky, R. and Leibler, S., Phys. Rev. Lett. 56, 2541 (1986).

10. For interfaces, one has $d_{\perp}=1$, but vortex lines in 3-dimensional type-II superconductors have $\boldsymbol{d}_{1}=2$ and exhibit similar interactions, see Nelson, D. R., Phys. Rev. Lett. 60, 1973 (1988); Nattermann, T. and Lipowsky, R., Phys. Rev. Lett. 61, 2508 (1988)

11. Throughout this paper, temperature $T$ is measured in units of the Boltzmann constant

12. Similar arguments have been applied to the stretching of polymers and to the confinement of polymers in pores, see Ref [4].

13. Lipowsky, R. and Baumgärtner, A., Phys. Rev. A (in press).

14. Maggs, A., Huse, D. A. and Leibler, S., (to be published) have used similar arguments for the adsorption of semiflexible polymers.

15. For linear polymers in $\boldsymbol{d}=\mathbf{1}+\boldsymbol{d}_{\boldsymbol{k}}$, Flory's well-known estimate is $v \simeq 3 /(d+3)$

16. Kantor, Y., Kardar, M. and Nelson, D. R., Phys. Rev. A35, 3056 (1987) found from a Flory-type argument that $v \simeq 4 / 5$ for self-avoiding tethered membranes in $\boldsymbol{d}=\mathbf{2}+\mathrm{L}$ However, recent computer simulations by F. F. Abraham, W. E. Rudge, and M. Plischke, Phys. Rev. Lett. 62, 1757 (1989) and by J.-S. Ho and A. Baumgärtner (to be published) indicate that self-avoidance always leads to an uncrumpled state of tethered membranes with $v=1$

17. For a crumpled manifold, $\tau$ is equal to the fractal dimensionality of the manifold.

18. The fluctuation-induced interaction also shows up in dynamic processes. [1] For example, a simple relaxational dynamics, $\partial l \partial t \sim-\partial V_{\mathrm{FL}} / \partial l$ leads to the time-dependent separatin $l(t) \sim t^{\theta}$ of the manifolds with $\theta=1 /(2+\tau)$
19. Kroll, D. M., Lipowsky, R. and Zia, R. K. P., Phys. Rev. B32, 1862 (1985); and Zia, R. K. P., Lipowsky, R. and Kroll, D. M., Am. J. Phys. 56, 160 (1988)

20. See, e.g., Wu, X., Schlossman, M. and Franck, C., Phys. Rev. B33, 402 (1986)

21. See, e.g., Zhu, D.-M. and Dash, J. G., Phys. Rev. Lett. 60, 432 (1988)

22. Zhu, D.-M., Pengra, D. and Dash, J. G., Phys. Rev. B37, 5586 (1988)

23. Lipowsky, R. and Nieuwenhuizen, T. M., J. Phys. A21, L89 (1988).

24. For wetting in $d=1+1$, see Abraham, D. B., Phys. Rev. Lett. 44, 1165 (1980)

25. For adhesion of fluid and crystalline membranes in $d=2+1$, see Lipowsky, R. and Leibler, S., In Ref. [9]; Lipowsky, R., Europhys. Lett. 7, 255 (1988)

26. For adsorption of polymers, see Eisenriegler, E., Kremer, K. and Binder, K., J. Chem. Phys. 77, 6296 (1982) and Ref. [13].

27. For adsorption of crumpled membranes, see Ref. [13]

28. Heilfrich, W. and Mutz, M., in Random Fluctuations and Growth (Edited by H. E. Stanley and N. Ostrowsky). Kluwer Academic Publishers, Dordrecht (1988). Mutz, M. and Helfrich, W., Phys. Rev. Lett. 62, 2881 (1989).

29. Binder, M. and Landan, D. P., Phys. Rev. B37, 1745 (1988).

30. Gomper, G. and Kroll, D. M., Phys. Rev. B38, 459 (1988).

31. Pluis, B., Denier van der Gon, A. W., Frencken, J. W. M. and van der Veen, J. F., Phys. Rev. Lett. 59, 2678 (1987).

32. Prince, K. C., Breuer, U. and Bonzel, H. P., Phys. Rev. Lett. 60, 1146 (1988).

33. Alvarado, S. F., Campagna, M., Fattah, A. and Uelhoff, W., Z. Phys. B66, 103 (1987)

34. Dosch, H., Mailaender, L., Lied, A., Peisl, J., Grey, F., Johnson, R. L. and Krummacher, S., Phys. Rev. Lett. 60, 2382 (1988)

35. Lipowsky, R., Phys. Rev. Lett 49, 1575 (1982); Lipowsky, R. and Speth, W., Phys. Rev. B28, 3983 (1983).

36. Lipowsky, R., Breuer, U., Prince, K. C. and Bonzel, H. P., Phys. Rev. Lett. 62, 913 (1989).

37. Wilson, K. G., Phys. Rev. B4, 3184 (1971).

38. Lipowsky, R. and Fisher, M. E., Phys. Rev. Lett. 57, 2411 (1986) Phys. Rev. B36, 2126 (1987).

39. Lipowsky, R., in Ref. [25],

40. Lipowsky, R., Phys. Rev. Lett 62, 704 (1989).

41. Wegner, F., in Phase Transitions and Critical Phenomena, Vol. 6 (Edited by C. Domb and M. S. Green). (Academic Press, London (1976)

42. Kosterlitz, J. M., J. Phys. C7, 1046 (1974)

43. In Ref. [40], $\bar{\omega}$ is denoted by $\omega / \zeta$

44. Thus, fluid membrane in $d=2+1$ should exhibit the same exponent $\psi$ as interfaces in $d=1+1$ since $\tau=2$ in both cases. This is consistent with recent Monte Carlo data on fluid membranes, see Lipowsky, R. and Zielinska, B., Phys. Rev. Lett. 62, 1572 (1989).

45. This scaling relation still holds in the SFL regime even though it is no longer useful to interpret $f_{\mathbf{s}}$ as a fluctuation-induced interaction. For the SFL regimes in $d=1+1$, one has $\tau \psi=2-\alpha=2$ and, thus, the specific heat exponent $\boldsymbol{\alpha}=\mathbf{0}$ for wetting in pure and in random-bond systems, and for adsorption of ideal and of selfavoiding polymers. 